\title{
Basis set effects on the energy and hardness profiles of the hydrogen fluoride dimer
}

\author{
MIQUEL TORRENT-SUCARRAT, MIQUEL DURAN, JOSEP M LUIS* and \\ MIQUEL SOLÀ* \\ Institut de Química Computacional and Departament de Química, Universitat de Girona, E-17071 \\ Girona, Catalonia, Spain \\ e-mail: miquel.sola@udg.es; josepm.luis@udg.es
}

\begin{abstract}
In earlier work, the present authors have shown that hardness profiles are less dependent on the level of calculation than energy profiles for potential energy surfaces (PESs) having pathological behaviors. At variance with energy profiles, hardness profiles always show the correct number of stationary points. This characteristic has been used to indicate the existence of spurious stationary points on the PESs. In the present work, we apply this methodology to the hydrogen fluoride dimer, a classical difficult case for the density functional theory methods.
\end{abstract}

Keywords. Ab initio calculations; density functional calculations; potential energy surface (PES); maximum hardness principle (MHP); spurious stationary points; hydrogen fluoride dimer.

\section{Introduction}

The concept of a Potential Energy Surface (PES) for molecules stems from the Born-Oppenheimer approximation for the separation of the nuclear and electronic motion. The PES is a smooth function that gives how the potential energy varies as relative coordinates of the atomic nuclei involved in a chemical system are changed. For a nonlinear molecule, consisting of $n$ atoms, the PES depends on $3 n-6$ coordinates corresponding to the total number of internal degrees of freedom. Years of experience have proven that the notion of the PES is extremely powerful in analysing molecular structure, spectroscopy, and chemical reactivity. Indeed, the whole of chemistry can be considered as a series of exercises on PESs. ${ }^{1,2}$

The characterization of the PES is normally accomplished by locating and characterizing the chemical relevant stationary points, i.e. minima and transition states, which normally implies an important computational effort. Moreover, it is not always feasible to fully reproduce the experimental results. This is particularly true in the case of chemical reactions, where the number and/or nature of stationary points (minima or transition states) may change with the basis set and/or the method of calculation. ${ }^{3-8}$ In these pathological systems, high level $a b$ initio methods

*For correspondence (not always computationally affordable) are required to be certain of the correctness of the calculated PES.

The contribution of the density functional theory $(\mathrm{DFT})^{9,10}$ in the knowledge of the PES has been very important. The nonlocal hybrids DFT methods provide results with accuracy comparable to or frequently even higher than results from ab initio correlated calculations and using less computer time. However, DFT methods also show pathological behaviors, the hydrogen fluoride (HF) dimer being a classical example in the literature. ${ }^{4-8}$

Both experiment and high level calculations indicate that the HF dimer has a planar $\left(C_{s}\right)$ equilibrium structure (see figure 1). One HF molecule forms a nearly linear hydrogen bond to the other. ${ }^{4,5}$ Moreover, the PES of HF dimer also contains a transition state with $C_{2 h}$ cyclic structure, that connects the two possible $C_{s}$ minima.

It is also know that DFT methods in conjunction with small basis sets tend to predict the cyclic

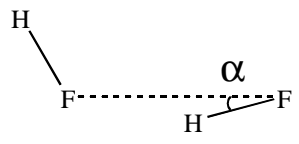

Minimum $C_{s}$

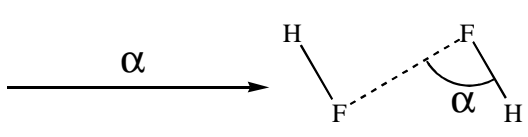

TS $C_{2 h}$
Figure 1. A schematic representation of the linear transit path obtained by changing the HFF angles in the HF dimer with their correct stationary points. 
structure as the only stable structure on the PES. ${ }^{6-8}$ This error can be solved when accounting for the basis set superposition error (BSSE) through the optimization process. $^{8}$

In a recent article, ${ }^{11}$ the present authors studied a series of well-established inter and intramolecular rearrangements, where the use of a weak methodology or a poor basis set results in the presence of spurious stationary points on the PESs. In that work, the hardness profiles were found to be less sensitive to the change of basis or/and method than the energy profiles. Furthermore, the hardness profiles always presented the correct number of stationary points. For these reasons, the present authors concluded that hardness profiles can be very useful in checking the validity of the energy profiles that owing to computational requirements cannot be treated with high level ab initio methods.

Hardness is a measure of the resistance of a chemical species to change its electronic configuration. ${ }^{12}$ The analytical definition of $\eta$ was given by Parr and Pearson ${ }^{13}$ in the framework of the conceptual DFT $^{14,15}$ as the second-order partial derivative of the total electronic energy, $E$, with respect to the total number of electrons, $N$, at a fixed external potential $\mathrm{v}(\mathbf{r})$ :

$$
\eta=\left(\partial^{2} E / \partial N^{2}\right)_{\mathrm{v}(\mathbf{r})}
$$

$\mathrm{V}(\mathbf{r})$ is the potential acting on an electron at $\mathbf{r}$ due to the nuclear attraction plus such other external forces as may be present. Related to the hardness, there are two fundamental chemical reactivity principles: the hard and soft acids and bases (HSAB) ${ }^{12}$ principle and the maximum hardness principle (MHP)..$^{12,16,17}$

The MHP affirms that systems tend to a state of maximum hardness at constant temperature, external potential, and chemical potential. ${ }^{17}$ Despite these strict constraints not being followed in any kind of nuclear displacement, it has been found that this principle holds in molecular vibrations, ${ }^{18}$ internal rotations, ${ }^{19}$ and different types of chemical reactions ${ }^{20}$ although some failures have also been reported. ${ }^{21,22}$ Gázquez et $a l^{23}$ demonstrated, using an approximate expression for the energy changes along the reaction path, that under conditions of constant chemical potential the hardness profile has a maximum (minimum) where the energy profile has a minimum (maximum). Although in a reaction profile the chemical potential is not constant, previous studies on hardness profiles ${ }^{18-21}$ indicate that a stationary point of the hardness is usually located close to a stationary point of the PES.

In a reaction which follows the MHP, the minima and transition states of the PES represent maxima and minima of the hardness profile respectively, although they do not exactly coincide at the same locations. In contrast in a reaction which breaks the MHP, the maxima and minima of the hardness profiles are respectively found near the transition states and minima of the PESs. Thus, the number of stationary points in the PESs and the hardness profiles usually become identical. Consequently, the presence of a different number of stationary points in the hardness and energy profiles is an indication of the presence of spurious stationary points on the PESs of the chemical processes.

The aim of the present work is to present another example of this property of the hardness profile. For this reason, we evaluate the energy and hardness profiles for the HF dimer at the B3LYP level, using eight different standard basis sets.

\section{Computational details}

The finite difference approximation and the Koopmans' theorem $^{24}$ in (1) lead to the following working definitions of the hardness:

$$
\begin{aligned}
& \eta_{1}=I-A \text { and } \\
& \eta_{2}=\varepsilon_{\text {LUMO }}-\varepsilon_{\text {HOMO }}
\end{aligned}
$$

where $I$ and $A$ are the first vertical ionization potential and electron affinity of the neutral molecule respectively, and $\varepsilon_{\mathrm{LUMO}}$ and $\varepsilon_{\mathrm{HOMO}}$ are the energies of the lowest unoccupied molecular orbital and the highest occupied molecular orbital respectively.

To construct the PES of the HF dimer a linear transit path is chosen, which is obtained by freezing the HFF angle at differing values and optimizing the rest of internal coordinates (see figure 1). The negative and positive values of the HFF angle in figures 2 and 3 are related to the HFFH dihedral angles of 0 and $180^{\circ}$ respectively. The nature of stationary points has always been checked by computing their harmonic vibrational frequencies. At each optimized point of the linear transit path, hardness and energy have been evaluated to depict their corresponding profiles. An alternative choice to the calculated linear transit path could be to use the intrinsic reaction path, IRP, although for chemical systems with shallow 
(a)

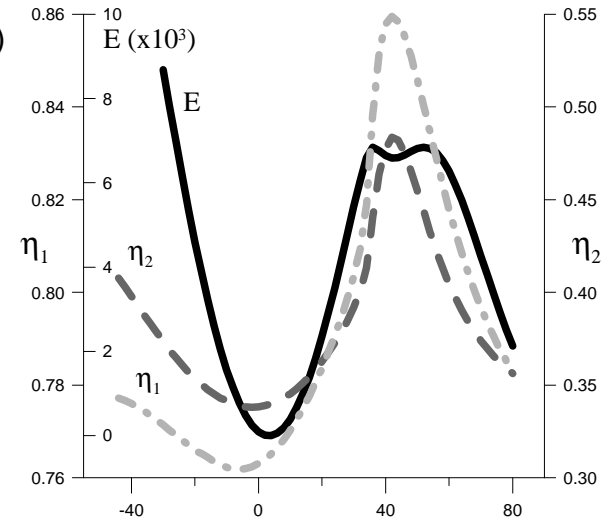

(c)

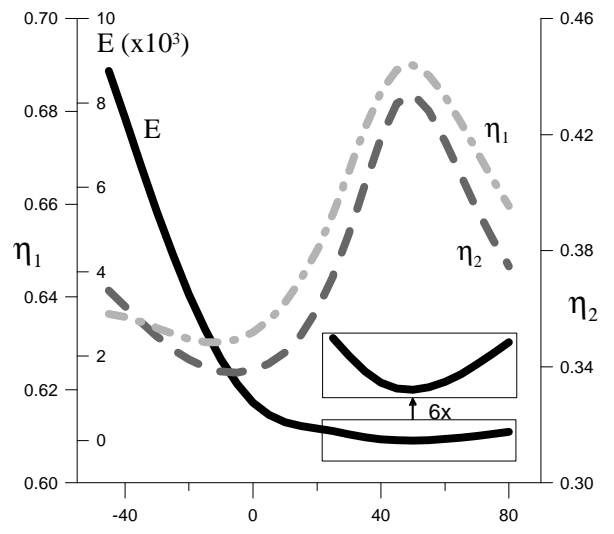

(e)

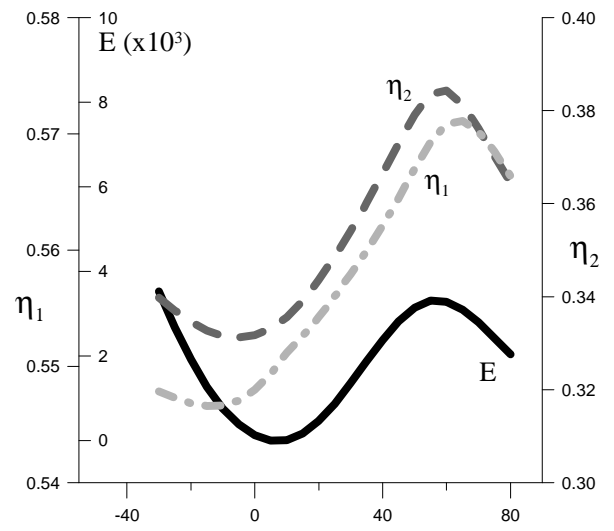

(g)

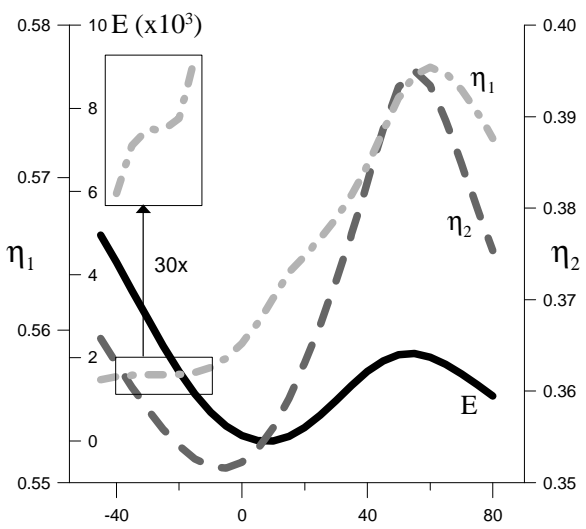

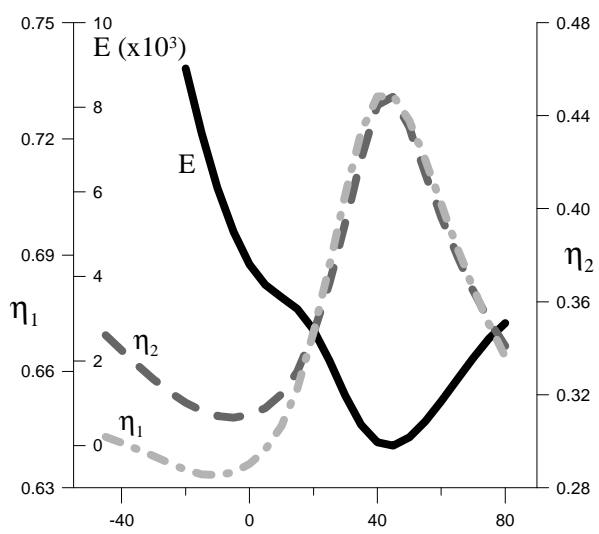

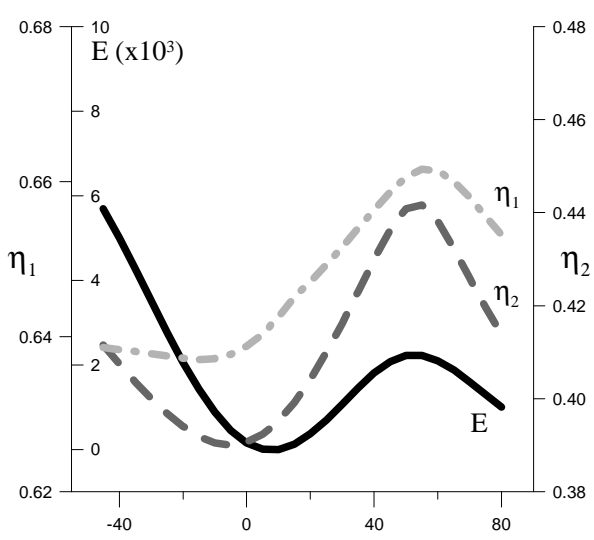

(d)
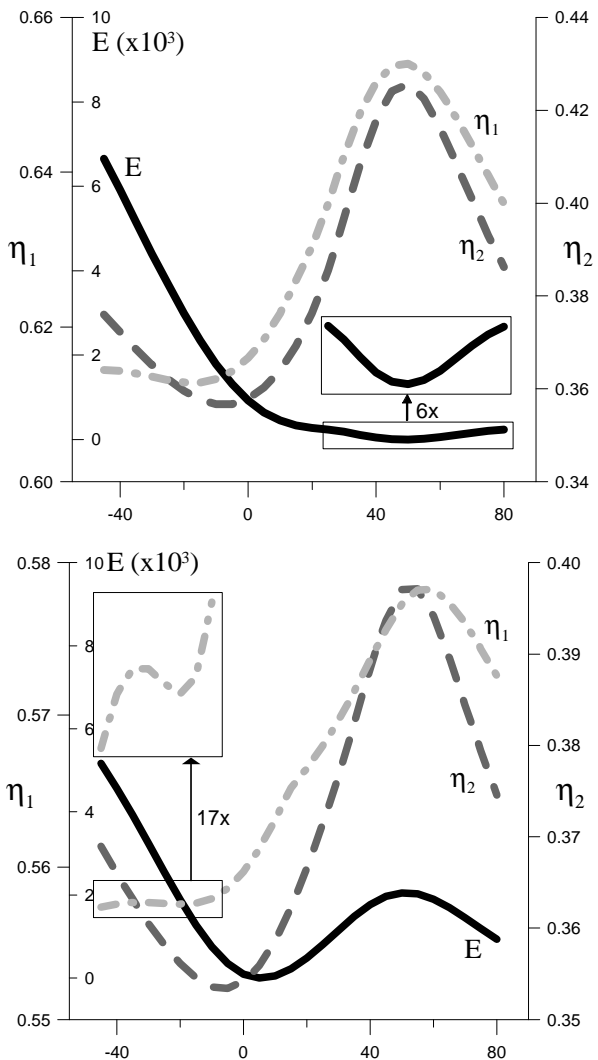

(b)

Figure 2. B3LYP profiles of the relative energies (solid line) and hardness ( $\eta_{1}$ dotted-dashed line and $\eta_{2}$ dashed line) for the linear transit path described by the HFF angle calculated with the basis sets: (a) STO-3G, (b) 3-21G, (c) 6-31G, (d) 6-31+G(d), (e) 6-311++G, (f) 6-311G $(d, p),(\mathbf{g}) 6-311++\mathrm{G}(d, p)$, and (h) 6-311++G(3df, 3pd). The hardness $\eta_{1}$ and $\eta_{2}$ are calculated from (2) and (3), respectively. Hardness values and relative energies are given in atomic units. 
minima on the PES, like the HF dimer, the current implementations to follow the IRP have serious problems in connecting the TS with the minima.

Geometry optimizations and hardness calculations have been performed with the B3LYP method ${ }^{25}$ using a series of Pople standard basis sets. ${ }^{26}$ The energies of the cationic and anionic doublet species, needed to obtain the vertical $I$ and $A$, have been computed within the unrestricted methodology at the geometry of the neutral system. All these calculations have been carried out with the Gaussian 98 package. ${ }^{27}$

\section{Results and discussion}

Figure 2 displays the $E, \eta_{1}$, and $\eta_{2}$ profiles for the linear transit path described by the HFF angle computed at the B3LYP level using the STO-3G, 3$21 \mathrm{G}, 6-31 \mathrm{G}, 6-31+\mathrm{G}(\mathrm{d}), 6-311++\mathrm{G}, 6-311 \mathrm{G}(d, p)$, $6-311++\mathrm{G}(d, p)$, and $6-311++\mathrm{G}(3 d f, 3 p d)$ basis sets. The best $a b$ initio estimates ${ }^{7}$ of the potential energy profile show a minimum for an HFF angle of about $7^{\circ}$ (experimental value of $\left.10 \pm 6^{\circ}\right)^{5}$ and a TS with cyclic structure $\left(C_{2 h}\right)$ of about $50^{\circ} .^{8}$ These two stationary points present an energy barrier of only $1.3 \mathrm{kcal} / \mathrm{mol}$ at the B3LYP/6-311++G $(3 d f, 3 p d)$ level. This proper energy profile has been well reproduced by the 6 $31+\mathrm{G}(d), 6-311++\mathrm{G}, 6-311++\mathrm{G}(d, p)$, and $6-311++\mathrm{G}$ $(3 d f, 3 p d)$ basis sets. In contrast, the $3-21 \mathrm{G}, 6-31 \mathrm{G}$, and $6-311 \mathrm{G}(d, p)$ PESs only present one spurious minimum at the $C_{2 h}$ cyclic structure, showing the well-known importance of the diffuse functions to accurately describe the hydrogen-bonded complex of the HF dimer. ${ }^{4,8}$ Finally, the STO-3G PES predicts four stationary points instead of two. In contrast to the $3-21 \mathrm{G}$ and $6-31 \mathrm{G}$ basis sets, the STO-3G basis set characterizes the minimum at $5^{\circ}$. However, it fails at the cyclic structure, predicting a spurious minimum at about $42^{\circ}$ and two false TSs very close to the $C_{2 h}$ geometry.

In contrast to the strong dependence of the PES on the basis set, the shape of the hardness is nearly independent of the basis set. Between the different basis sets, there are some important changes in the absolute values of the hardness, although their shapes along the linear transit path remain unchanged.

The $\eta_{2}\left(\varepsilon_{\text {LUMO }}-\varepsilon_{\text {HOMO }}\right)$ profiles in figure 2 for the eight basis sets studied present a minimum and a maximum at about $-5^{\circ}$ and $50^{\circ}$ respectively, indicating the existence of two stationary points in the PES. Thus, the difference between the number of stationary points of the energy and hardness profiles [in the STO-3G, 3-21G, 6-31G, and 6-311G $(d, p)$ basis sets] denotes the presence of spurious stationary points on the corresponding PESs. It is worth nothing that this intermolecular conformational change breaks the MHP, because the maximum and minimum of the hardness profile are respectively found near the TS and the minimum of the correct PES. This behavior can be understood by analyzing the variation of the HOMO and LUMO energies along the linear transit path. The maximum hardness near the cyclic structure is due to a larger stabilization of the HOMO energy and a destabilization of the LUMO energy, while the minimum structure presents the most unstable and most stable HOMO and LUMO energies respectively, along the rearrangement reaction. In contrast to previous cases, ${ }^{22}$ the behavior of the HOMO and LUMO energies along the linear transit path cannot be easily discussed from the changes in the shape of the orbitals.

The $\eta_{1}(I-A)$ profiles mimic the shape of the $\eta_{2}$ profiles, indicating the existence of two stationary points in the PESs with the only remarkable difference that the minimum of $\eta_{1}$ appears some degrees shifted to the left side, more or less at $-15^{\circ}$. However, there are two basis sets, $6-311++\mathrm{G}(d, p)$ and $6-311++\mathrm{G}$ $(3 d f, 3 p d)$, which show the expected minimum (in figure $2 \mathrm{~g}$ the minimum is very shallow), but then, the $\eta_{1}$ shape decreases creating a spurious maximum of hardness (see the insets of figures $2 \mathrm{~g}$ and $2 \mathrm{~h}$ ). The problem of the $\eta_{1}$ profiles arises from the evaluation of the energy of the system with $N-1$ electrons, because for the (HF $)_{2}^{+}$species there are two quasidegenerate electronic states $\left({ }^{2} A^{\prime}\right.$ and $\left.{ }^{2} A^{\prime \prime}\right)$, with a crossing point in the middle of the linear transit path. Therefore, the correct calculation of the ionization potential of this dimer should be carried out using multiconfiguration methods. Despite this multiconfiguration character of the ionization potential energy, the $\eta_{1}$ profiles of figure 2 have always been calculated taking the most stable electronic state at the B3LYP level. Thus, the $\eta_{1}$ results should be viewed with some skepticism. Obviously, this problem could be overcome using a multiconfiguration method like MCSCF, but this is out of the scope of this paper. In contrast, the neutral HF dimer shows HOMO and HOMO-1 orbitals close in energy, but along the linear transit path there is no interchange of these orbitals. Therefore, in this case, the $\eta_{2}$ profiles should be considered of better quality, in principle, than the $\eta_{1}$ profiles. 


\section{Conclusions}

In this work, we have studied the energy and hardness profiles for the hydrogen fluoride dimer using the B3LYP level and eight different basis sets. In an analogous manner to our previous work, we have found that the hardness profile is more basis set-independent than the energy profile. Indeed, the shape of the hardness profile is qualitatively the same for all basis set analyzed, while the energy profile is wrong with the STO-3G, 3-21G, 6-31G, and 6-311G $(d, p)$ basis sets. Therefore, we have confirmed that, for the hydrogen fluoride dimer, the hardness profiles can also be used to check the validity of the energy profiles. With the larger basis sets, the hardness calculated using the approximation $I-A\left(\eta_{1}\right)$ does not reproduce the shape of the $\varepsilon_{\text {LUMO }}-\varepsilon_{\text {HOMO }}\left(\eta_{2}\right)$. The $\eta_{1}$ profiles contain a spurious maximum of hardness, probably due to the multiconfiguration character of the ground state of the $N-1$ system.

\section{Acknowledgements}

Support for this work from the Spanish Ministerio de Ciencia y Tecnología, from the DURSI (Generalitat de Catalunya), and the use of the computational facilities of the Catalonia Supercomputer Center (CESCA) are gratefully acknowledged. MS thanks the DURSI for financial support through the Distinguished University Research Promotion, 2001. We are grateful to Dr. Pedro Salvador for helpful comments.

\section{References}

1. Schlegel H B 1987 Ab initio methods in quantum chemistry (ed.) K P Lawley (New York: John Wiley \& Sons)

2. Cramer C J 2002 Essentials of computational chemistry (New York: John Wiley \& Sons)

3. (a) Cremer D 1978 J. Chem. Phys. 69 4440; (b) Cremer D 1978 J. Chem. Phys. 69 4456; (c) Grev R S and Schaefer H F III 1984 J. Chem. Phys. 82 4126; (d) Szczesniak M M, Scheiner S and Bouteiller Y $1984 \mathrm{~J}$. Chem. Phys. 81 5024; (e) Flaud J-M, Camy-Peyret C, Johns J W C and Carli B 1989 J. Chem. Phys. 91 1504; (f) Shen M, Xie Y, Schaefer H F III and Deakyne C A 1990 J. Chem. Phys. 93 3379; (g) Turi L and Dannenberg J J 1993 J. Phys. Chem. 97 7899; (h) Ma N L, Wong S S, Paddon-Row M N and Li W-K 1993 Chem. Phys. Lett. 213 189; (i) Simon S, Duran M and Dannenberg J J 1996 J. Chem. Phys. 105 11024; (j) Nielsen I M B, Allen W D, Császár A G and Schaefer HF III 1997 J. Chem. Phys. 107 1195; (k) Re S, Osamura Y, Suzuki Y and Schaefer H F III 1998 J.
Chem. Phys. 109 973; (1) Tsuzuki S, Uchimaru T, Mikami M and Tanabe K 1998 J. Chem. Phys. 109 2169; (m) Valeev E F and Schaefer H F III $1998 \mathrm{~J}$. Chem. Phys. 108 7197; (n) Kisiel Z, Pietrewicz B A, Fowler P W, Legon A C and Steiner E 2000 J. Phys. Chem. A104 6970; (o) Salvador P, Simon S, Duran M and Dannenberg J J 2000 J. Chem. Phys. 113 5666; (p) Li Z-H and Fan K-N 2002 J. Phys. Chem. A106 6659; (q) Tommunphean S, Parasuk V and Karpfen A 2002 J. Phys. Chem. A106 438; (r) Wang X-B, Yang X, Wang L-S and Nicholas J B 2002 J. Chem. Phys. 116 561; (s) Giesen D J and Phillips J A 2003 J. Phys. Chem. A107 4009; (t) Fiacco D L and Leopold K R 2003 J. Phys. Chem. A107 2808

4. Maerker C, Schleyer P $\mathrm{v}$ R, Liedl K R, Ha T-K, Quack M and Suhm M A 1997 J. Comput. Chem. 18 1695

5. Howard B J, Dyke T R and Klemperer W 1984 J. Chem. Phys. 815417

6. Del Bene J E, Pearson W B and Szczepaniak K 1995 J. Phys. Chem. 9910705

7. Hobza P, Sponer J and Reschel R 1995 J. Comput. Chem. 111315

8. Salvador P, Fradera X and Duran M 2000 J. Chem. Phys. 11210106

9. Parr R G and Yang W 1989 Density-functional theory of atoms and molecules (New York: Oxford University Press)

10. Koch W and Holthausen M C 2000 Chemist's guide to density functional theory (Weinheim: Wiley-VCH)

11. Torrent-Sucarrat $\mathbf{M}$, Luis $\mathbf{J} \mathbf{M}$, Duran M and Solà M 2004 J. Chem. Phys. 2310914

12. Pearson R G 1997 Chemical hardness: Applications from molecules to solids (Weinheim: Wiley-VCH)

13. Parr R G and Pearson R G 1983 J. Am. Chem. Soc. 1057512

14. Chermette H 1999 J. Comput. Chem. 20129

15. Geerlings P, De Proft F and Langenaeker W 2003 Chem. Rev. 1031793

16. (a) Pearson R G 1987 J. Chem. Educ. 64 561; (b) Pearson R G 1999 J. Chem. Educ. 76267

17. Parr R G and Chattaraj P K 1991 J. Am. Chem. Soc. 1131854

18. (a) Pearson R G and Palke W E 1992 J. Phys. Chem. 96 3283; (b) Makov G 1995 J. Phys. Chem. 99 9337; (c) Pal S, Vaval N and Roy R 1993 J. Phys. Chem. 97 4404; (d) Chattaraj P K, Fuentealba P, Jaque P and Toro-Labbé A 1999 J. Phys. Chem. A103 9307

19. (a) Cárdenas-Jirón G I, Lahsen J and Toro-Labbé A 1995 J. Phys. Chem. 99 5325; (b) Cárdenas-Jirón G I and ToroLabbé A 1995 J. Phys. Chem. 99 12730; (c) CárdenasJirón G I, Gutiérrez-Oliva S, Melin J and Toro-Labbé A 1997 J. Phys. Chem. A101 4621; (d) Cárdenas-Jirón G I and Toro-Labbé A 1997 J. Mol. Struct. (Theochem.) 390 79; (e) Gutiérrez-Oliva S, Letelier J R and Toro-Labbé A 1999 Mol. Phys. 96 61; (f) Uchimaru T, Chandra A K, Kawahara S, Matsumura K, Tsuzuki S and Mikami M 2001 J. Phys. Chem. A105 1343; (g) Parthasarathi R, Padmanabhan J, Subramanina V, Maiti B and Chattaraj P K 2003 J. Phys. Chem. A107 10346; (h) Cadet J, Grand A, Morell C, Letelier J R, Moncada J L and Toro-Labbé 
A 2003 J. Phys. Chem. A107 5334; (i) Chattaraj P K, Gutiérrez-Oliva S, Jaque P and Toro-Labbé A 2003 Mol. Phys. 101 2841; (j) Gutiérrez-Oliva S and Toro-Labbé A 2004 Chem. Phys. Lett. 383435

20. (a) Datta D 1992 J. Phys. Chem. 96 2409; (b) Kar T and Scheiner S 1995 J. Phys. Chem. 99 8121; (c) Chattaraj P K, Cedillo A, Parr R G and Arnett E M 1995 J. Org. Chem. 60 4707; (d) Ghanty T K and Ghosh S K 1996 J. Phys. Chem. 100 12295; (e) Toro-Labbé A 1999 J. Phys. Chem. A103 4398; (f) Pérez P and Toro-Labbé A 2000 J. Phys. Chem. A104 1557; (g) Chattaraj P K, Fuentealba P, Gómez B and Contreras R 2000 J. Am. Chem. Soc. 122 348; (h) Hohm U 2000 J. Phys. Chem. A104 8418; (i) Jaque P and Toro-Labbé A 2000 J. Phys. Chem. A104 995; (j) Jaque P and Toro-Labbé A 2002 J. Chem. Phys. 117 3208; (k) Ghanty T K and Ghosh S K 2002 J. Phys. Chem. A106 4200

21. (a) Kar T, Scheiner S and Sannigrahi A B 1998 J. Phys. Chem. A102 5967; (b) Solà M and Toro-Labbé A 1999 J. Phys. Chem. A103 8847; (c) Nguyen L T, Le T N, De Proft F, Chandra A K, Langenaeker W, Nguyen M T and Geerlings P 1999 J. Am. Chem. Soc. 121 5992; (d) Nguyen L T, De Proft F, Nguyen M T and Geerlings P 2001 J. Org. Chem. 66 4316; (e) Sicilia E, Russo N and Mineva T 2001 J. Phys. Chem. A105 442; (f) Gómez B,
Chattaraj P K, Chamorro E, Contreras R and Fuentealba P 2002 J. Phys. Chem. A106 11227; (g) Gómez B, Fuentealba P and Contreras R 2003 Theor. Chem. Acc. 110 421; (h) Blancafort Ll, Torrent-Sucarrat M, Luis J M, Duran M and Solà M 2003 J. Phys. Chem. A107 7337; (i) Torrent-Sucarrat M, Duran M, Luis J M and Solà M 2005 J. Phys. Chem. A109 615

22. (a) Torrent-Sucarrat M, Luis J M, Duran M and Solà M 2001 J. Am. Chem. Soc. 123 7951; (b) Gómez B, Chattaraj P K, Chamorro E, Contreras R and Fuentealba P 2002 J. Phys. Chem. A106 11227; (c) TorrentSucarrat M, Luis J M, Duran M and Solà M $2002 \mathrm{~J}$. Chem. Phys. 11710561

23. Gázquez J L, Martínez A and Méndez F 1993 J. Phys. Chem. 974059

24. Koopmans T 1934 Physica (Utrecht) 1104

25. (a) Lee C, Yang, W and Parr R G 1988 Phys. Rev. B37 785; (b) Becke A D 1993 J. Chem. Phys. 98 5648; (c) Stephens P J, Devlin F J, Chabalowski C F and Frisch M J 1994 J. Phys. Chem. 9811623

26. Hehre W J, Radom L, Schleyer P v R and Pople J A 1986 Ab initio molecular orbital theory (New York: Wiley)

27. Frisch M J et al 1998 Gaussian 98, revision A.11, Gaussian Inc., Pittsburgh, PA 